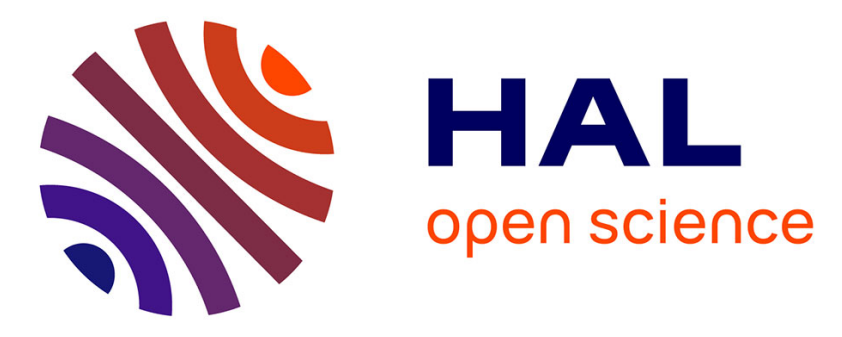

\title{
Fluorescence and spin properties of defects in single digit nanodiamonds
}

Julia Tisler, Gopalakrischnan Balasubramanian, Boris Naydenov, Roman

Kolesov, Bernhard Grotz, Rolf Reuter, Jean-Paul Boudou, Patrick A. Curmi, Mohamed Sennour, Alain Thorel, et al.

\section{To cite this version:}

Julia Tisler, Gopalakrischnan Balasubramanian, Boris Naydenov, Roman Kolesov, Bernhard Grotz, et al.. Fluorescence and spin properties of defects in single digit nanodiamonds. ACS Nano, 2009, 3 (7), pp.1959-1965. 10.1021/nn9003617 . hal-00481219

\section{HAL Id: hal-00481219}

https://hal-mines-paristech.archives-ouvertes.fr/hal-00481219

Submitted on 10 Jul 2018

HAL is a multi-disciplinary open access archive for the deposit and dissemination of scientific research documents, whether they are published or not. The documents may come from teaching and research institutions in France or abroad, or from public or private research centers.
L'archive ouverte pluridisciplinaire HAL, est destinée au dépôt et à la diffusion de documents scientifiques de niveau recherche, publiés ou non, émanant des établissements d'enseignement et de recherche français ou étrangers, des laboratoires publics ou privés. 


\title{
Fluorescence and Spin Properties of Defects in Single Digit Nanodiamonds
}

\author{
Julia Tisler, ${ }^{\dagger}$ Gopalakrishnan Balasubramanian, ${ }^{\dagger}$ Boris Naydenov, ${ }^{\dagger}$ Roman Kolesov, $^{\dagger}$ Bernhard Grotz, $^{\dagger}$ \\ Rolf Reuter, ${ }^{\dagger}$ Jean-Paul Boudou, ${ }^{\ddagger}$ Patrick A. Curmi, ${ }^{\ddagger}$ Mohamed Sennour, ${ }^{\S}$ Alain Thorel, ${ }^{\S}$ Michael Börsch, ${ }^{\dagger}$ \\ Kurt Aulenbacher," Rainer Erdmann, ${ }^{\perp}$ Philip R. Hemmer, ${ }^{\emptyset}$ Fedor Jelezko, ${ }^{\dagger}$ and Jörg Wrachtrup ${ }^{\dagger, *}$ \\ ${ }^{\dagger}$ Institute of Physics, University of Stuttgart, Stuttgart, Germany, ${ }^{\ddagger}$ Structure and Activity of Normal and Pathological Biomolecules, INSERM/UEVE U829, Bat. \\ Maupertuis, rue du père Jarlan 91025 Evry, France, ${ }^{\S}$ Centre des Matériaux, Mines-ParisTech, UMR7633 BP 87, 91003 Evry Cedex, France, UJohannes Gutenberg-Universität \\ Mainz, Johann-Joachim-Becher-Weg 45, D-55099 Mainz, Germany, ${ }^{\perp}$ PicoQuant GmbH, Rudower Chaussee 29, D-12489 Berlin, Germany, and "Texas A\&M University, \\ College Station, Texas 77843
}

L uminescent single-nanometer-sized diamond-like carbon particles have received considerable attention in biophysics, ${ }^{1-3}$ material science, ${ }^{3}$ nanomedicine, ${ }^{4}$ and photonics. ${ }^{5,6}$ Partly this is related to their extraordinary material properties, such as chemical inertness, biocompatibility, and hardness, and also partly due to their exceptional photostability. ${ }^{7,9}$ All of these applications desire the availability of sub-10 $\mathrm{nm}$ particles that show bright and stable photoluminescence. ${ }^{9}$ In addition, for applications such as drug delivery, biolabeling, and photonics, preventing the aggregation of small nanoparticles is also an important concern. ${ }^{8}$

So far, two different approaches have been taken to generate monodispersed, nanometer-sized carbon-based particles with bright photoluminescence. In one approach, laser ablation is used to produce photoluminescent carbon nanostructures. The resulting luminescence, however, is only observed upon proper surface passivation, ${ }^{10}$ and it is thus believed to be related to surface defects. One particular photoluminescent center in diamond, the nitrogenvacancy (NV) center, with its associated electron spin is recently receiving considerable attention due to potential applications in quantum optics, ${ }^{11-13}$ nanoscale magnetometry, ${ }^{14}$ and biology. ${ }^{14}$ Besides providing a spin label for magnetic-field-based nanoscale imaging, NV diamond nanocrystals have demonstrated exceptional stability as photoluminescent biocompatible labels. However, for long-term stability and maximum flexibility in functionalization, it is preferable to have photoluminescent defects (or color centers) that are incorporated inside diamond nanocrystals. Indeed, by
ABSTRACT This article reports stable photoluminescence and high-contrast optically detected electron spin resonance (ODESR) from single nitrogen-vacancy (NV) defect centers created within ultrasmall, disperse nanodiamonds of radius less than $4 \mathrm{~nm}$. Unexpectedly, the efficiency for the production of NV fluorescent defects by electron irradiation is found to be independent of the size of the nanocrystals. Fluorescence lifetime imaging shows lifetimes with a mean value of around $17 \mathrm{~ns}$, only slightly longer than the bulk value of the defects. After proper surface cleaning, the dephasing times of the electron spin resonance in the nanocrystals approach values of some microseconds, which is typical for the type Ib diamond from which the nanoparticle is made. We conclude that despite the tiny size of these nanodiamonds the photoactive nitrogen-vacancy color centers retain their bulk properties to the benefit of numerous exciting potential applications in photonics, biomedical labeling, and imaging.

KEYWORDS: fluorescent nanoparticle $\cdot$ defects in diamond $\cdot$ single molecule spectroscopy · confocal fluorescence microscopy · fluorescence lifetime imaging $\cdot$ electron spin resonance $\cdot$ single spin manipulation

freeing up the diamond surface for functionalization, it is possible to graft a variety of different biomolecules such as proteins or DNA onto nanodiamond surfaces. ${ }^{15-17}$ In addition to the NV center, which emits around $700 \mathrm{~nm}$, a variety of other bright luminescent defects have been generated in diamond nanocrystals (e.g., Ni impurities with fluorescence emission around 800 $\mathrm{nm}){ }^{18}$

Diamond nanocrystals have been produced with a size range down to about 4 $\mathrm{nm} .{ }^{19}$ However, up until now, photoluminescence of color centers has only been demonstrated down to $20 \mathrm{~nm}$ sized single nanocrystals. Attempts to observe stable luminescence from very small $5 \mathrm{~nm}$ nanodiamonds synthesized by explosive growth have so far only been successful for aggregates but not for single nanodiamonds. ${ }^{20}$ This raised the question of whether stable photoluminescence defects are observable at all in single nanometer-sized nanocrystals and if so how much lower would be the
*Address correspondence to
j.wrachtrup@physik.uni-stuttgart.de.

Received for review April 9, 2009 and accepted June 18, 2009.

Published online July 10, 2009. 10.1021/nn9003617 CCC: \$40.75

(c) 2009 American Chemical Society 

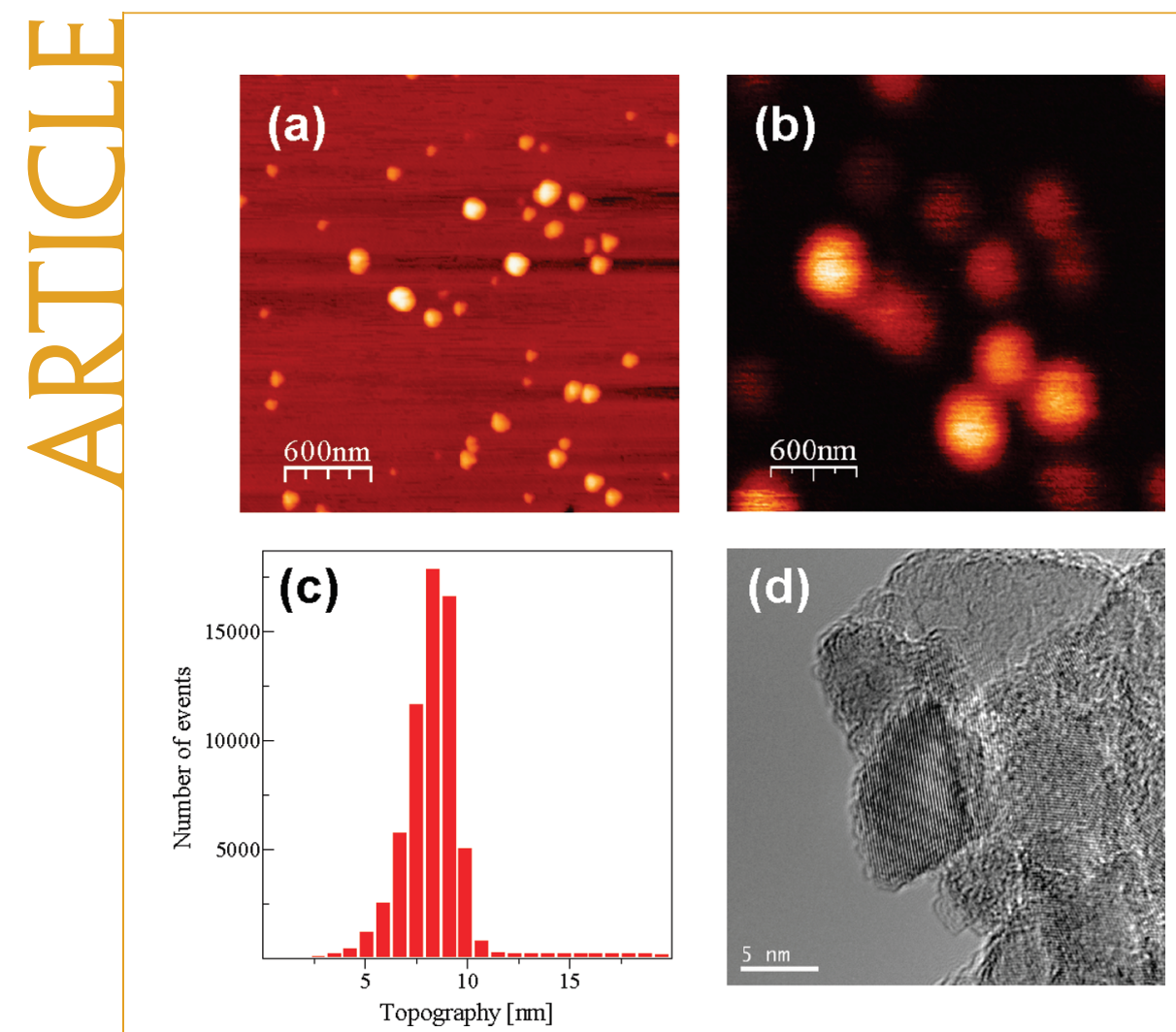

Figure 1. Characterization of irradiated diamond nanocrystals containing NV centers. (a) AFM scan showing the topography. (b) Confocal fluorescence micrograph showing most of the nanodiamonds are fluorescing. (c) Histogram of size distribution showing the mean particle size to be about 7.5-8 $\mathrm{nm}$. (d) HRTEM micrograph of small nanodiamonds; the diamond lattice is clearly visible.

yield for color center generation in such small crystallites. Here we show that down to a size of about $5 \mathrm{~nm}$ the fluorescence properties of NV color centers in diamond nanocrystals remain unaltered, as do their spin properties, and furthermore, they can be produced with similar yield to bulk crystals. These observations are critical for the use of nanocrystals in frontier applications where sizes below $10 \mathrm{~nm}$ are required.

For our investigations, we used commercially available diamond nanocrystals (Syp 0.02 van Moppes SA) with an average size of $10 \mathrm{~nm}$. These nitrogen-rich nanocrystals were irradiated with electrons and upon annealing produced stable NV defect centers. Electron microscopy of the nanocrystals as well as AFM-based measurements of nanocrystals dispersed on a coverslip (see Figure 1) confirms the size range and shows a significant fraction of crystals with sizes below $10 \mathrm{~nm}$. (Refer to Materials and Methods for details.)

\section{RESULTS AND DISCUSSION}

The fluorescence properties of the nitrogen-vacancy (NV) diamond color centers have been particularly intensely investigated owing to their strong optical transition, high fluorescence quantum yield, exceptional photostability, and spin properties..$^{21}$ It was shown that NV defects can be introduced in nitrogen containing diamond lattices by creation of vacancies and subsequent annealing at temperatures above $700{ }^{\circ} \mathrm{C}$. Ion im- plantation was proven to be very efficient for generation of vacancies;' however, the narrow stopping range of ions limits the thickness of the layer where maximum damage is produced. For nanocrystals, a better method of producing vacancies is high energy electron irradiation. The cross section for electron-carbon collision depends on their mass ratio. Hence electrons have a low probability to knock out carbons and instead lose most of their energy due to dissipative ionization processes. As a result, the electron penetration depth is large, and uniform damage can be created in thick samples. Though the procedure of creating NV centers is known, the key to produce stable fluorescence in small nanodiamonds is to select material with sufficient nitrogen atoms in substitution form and irradiating with high energy electrons to the right dosage. Furthermore, for ultrasmall crystals, surface properties are crucial. Therefore, we employed high vacuum $\left(10^{-6} \mathrm{mbar}\right)$ annealing followed by triacid treatment (refer to Materials and Methods).

Creating NV Defects in Nanodiamonds: For our experiments, nanodiamonds were irradiated with electrons of $8 \mathrm{MeV}$ energy to a dose of about $10^{20}$ electrons $/ \mathrm{cm}^{2}$. Published data show that a single $8 \mathrm{MeV}$ electron creates roughly three vacancies over its $10 \mathrm{~cm}$ stopping range in the diamond..$^{22}$ This results in roughly $3 \times 10^{19}$ vacancies per $\mathrm{cm}^{3}$ or 16 vacancies per spherical $10 \mathrm{~nm}$ diameter nanocrystal. The nitrogen concentration in our nanocrystals is 200 ppm based on electron spin resonance data, which corresponds to about 18 nitrogens per $10 \mathrm{~nm}$ diameter nanocrystal. From previous experiments in nitrogen-rich bulk diamond, we know that under these approximate conditions roughly $1 \mathrm{NV}$ defect is created per 100 vacancies, giving about $0.16 \mathrm{NV}$ per $10 \mathrm{~nm}$ diameter nanocrystal. A detailed analysis of AFM pictures like the one shown in Figure 1a and fluorescence images of the same area indicate that $35 \%$ of the nanocrystals contain a fluorescent defect center. We conclude that the production yield of NV defects in the nanocrystal is comparable to the yield in bulk material. This result might appear surprising at first hand because the vacancy annihilation process is expected to differ significantly for diamond nanocrystals and bulk material mostly because of the large surface to volume ratio. The probability of a vacancy to diffuse to the surface instead of binding to a nitrogen atom scales as $r^{2}$ ( $r$ is nanodiamond radius). ${ }^{20}$ Therefore, one expects a significantly lower yield for NV formation in small nanocrystals as compared to the bulk because the vacancies are mostly expected to anneal to the surface. The fact that this is not observed in our experiments suggests that the vacancies do not diffuse far before com- 
bining with a nitrogen or interstitial carbon created by the irradiation process and, therefore, do not have time to reach the surface.

Fluorescence Lifetime Studies: One of the key features of fluorescent defects in diamond nanocrystals is their unsurpassed photostability. Yet, as with all guest-host systems, photostability is expected to be a function of the host size. Hence it is particularly interesting to investigate the photophysics of defects in small nanocrystals. To begin with, we concentrate on fluorescence decay measurements of NV defects. In order to gain a statistically significant insight into the distribution of fluorescence lifetimes, we recorded a fluorescence lifetime image of our sample (see Figure 2a).

From these experiments, we determine nanocrystal fluorescence decay times between $\tau_{\mathrm{ND}}=10$ and $25 \mathrm{~ns}$ with an average of $17 \mathrm{~ns}$. The average decay time of NV defects in bulk diamond was determined to be around $\tau_{\mathrm{b}}$ $=12 \mathrm{~ns}^{23}$ close to the lower edge of the nanocrystal lifetime distribution. Fluorescence lifetime lengthening in nanocrystals has been described earlier ${ }^{11}$ and will be discussed here only briefly. The effect is attributed to the strong change in refractive index of the surrounding medium when going from bulk diamond to nanocrystals. This affects both the one-photon electric field and the mode density. Since the radiative rate is proportional to the product of these two quantities, the resulting radiative rate in a medium of refractive index $n$ is given by $\Gamma_{n}=n \Gamma_{0}$. Here $\Gamma_{0}$ is the radiative decay rate of the defect in vacuum. In this equation, we did not take into account any local field correction factors but rather assume that if there are any such corrections to the local field they are identical in nanocrystals and in the bulk. In order to derive the correct value for $n$ in the case of nanodiamonds, it is not the diamond refractive index of $n_{d}=2.4$ playing the key role but rather the refractive index of the coverslip the nanocrystals are deposited on. Given our sample geometry, a center placed inside a nanocrystal emits into air for one-half sphere and in glass $\left(n_{\mathrm{s}}=1.5\right)$ for the other half sphere. By using the above equation, one obtains an effective fluorescence decay rate of $\Gamma_{\text {average }}=1 / 2\left[1 / n_{\mathrm{d}} \tau_{\mathrm{b}}+n_{\mathrm{s}} / n_{\mathrm{d}} \tau_{\mathrm{b}}\right]{ }^{11}$ With the above values for $n$, a value for $\left(\Gamma_{\text {average }}\right)^{-1}=$ 22 ns results, which is somewhat longer but reasonably close to the average fluorescence lifetime found in experiments. From Figure 2, it is apparent that we do find a significant distribution of lifetimes. Part of this might be attributed to the presence (or absence) of surface quenchers. However, we believe that most of the graphite has been removed by surface treatment.

Rather we suggest that a significant fraction of the dis- the dipole orientation of the NV center with respect to the glass coverslip they are deposited on. Lukosz and Kunz calculated the dependence of the radiative decay rate to be $\Gamma_{\mathrm{av}}^{-1}=\cos \alpha^{2} \Gamma_{\mathrm{av} \perp}^{-1}$ $+\sin \alpha^{2} \Gamma_{\text {av }}^{-1} \cdot{ }^{24}$ This defines a periodically modulated function of the orientation angle $\alpha$ with respect to the surface. The amplitude of lifetime variation versus $\alpha$ for the present case is roughly 20 ns as determined from the width of the lifetime distribution in Figure $2 \mathrm{~b}$.

Significantly, we do not find a correlation of nanocrystal size and lifetime as shown in Figure $2 c$, where a large crystal with a $\tau_{\mathrm{ND}}$ of $9 \mathrm{~ns}$ and a small one with a $\tau_{\mathrm{ND}}$ of $25.1 \mathrm{~ns}$ is shown. Figure $3 \mathrm{a}-\mathrm{c}$ exemplifies a singledigit nanocrystal with a size of $7 \mathrm{~nm}$, which shows stable fluorescence and a lifetime of around 14 ns. This demonstrates that even single-digit crystals do support color centers with stable photoluminescence.

\section{Investigations on the Single Spin Properties of NV Defects in}

Nanocrystals: To complement our photoluminescence studies, we investigated the electron spin properties of NV defects in nanocrystals. As reported previously, the NV defect allows for the fluorescence detection of electron spin resonances in the ground (and excited) states. ${ }^{21}$ Owing to the possible application of NV defects in nanocrystals as sensitive and spatially highly resolving magnetic field detectors, their spin properties are of considerable interest. The $S=1$ spin state is governed by two contributions $H=D\left(S_{z}^{2}-S(S+1) / 3\right)+$ $E\left(S_{x}^{2}+S_{y}^{2}\right)+g \mu_{B} B S$ where $S$ is the electron spin, $D$ the zero field splitting tensor, $g$ the electron $g$-factor, $\mu_{B}$ the Bohr magneton, and $B$ the external magnetic field. The zero field splitting tensor $D$ arises from dipolar interaction of the two unpaired $S=1$ electron spins. Since the tensor is traceless, it can be described by two parameters usually labeled $D$ and $E$. $D$ measures the average distance of the two electron spins, while $E$ measures the

(b)

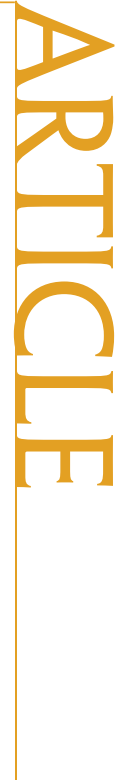

(c)
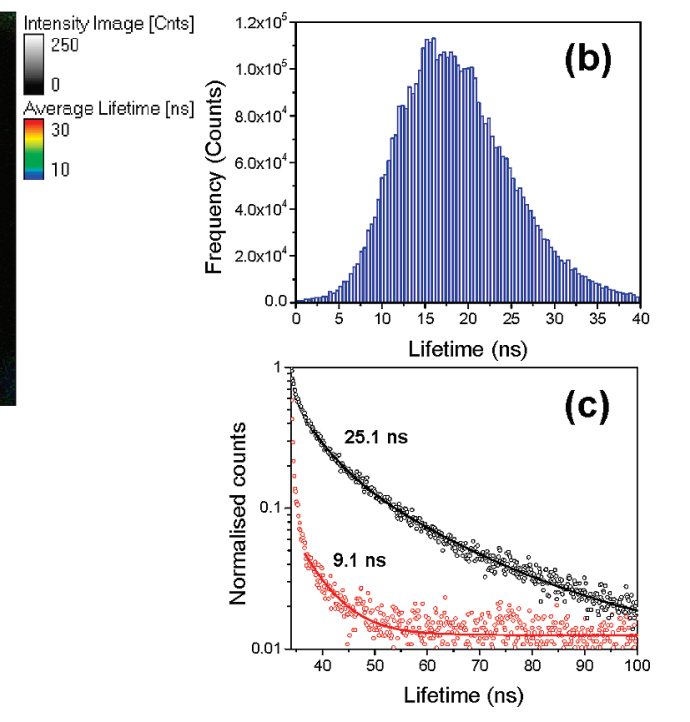
1

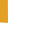




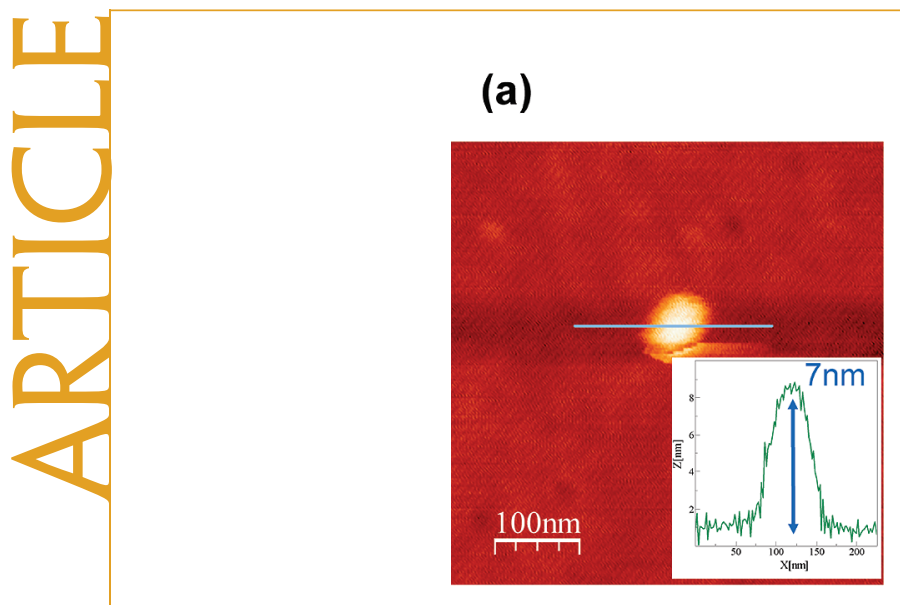

(b)

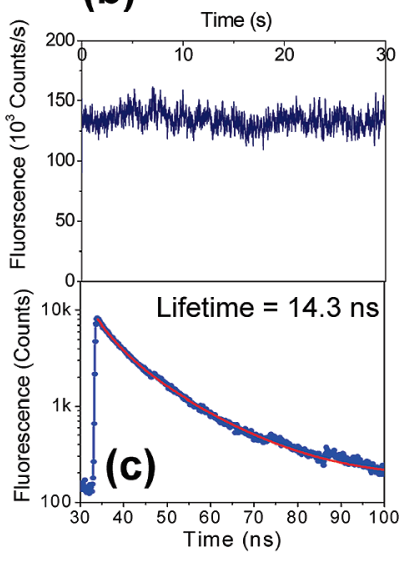

(d) $\mathrm{MW}$ frequency $(\mathrm{MHz})$

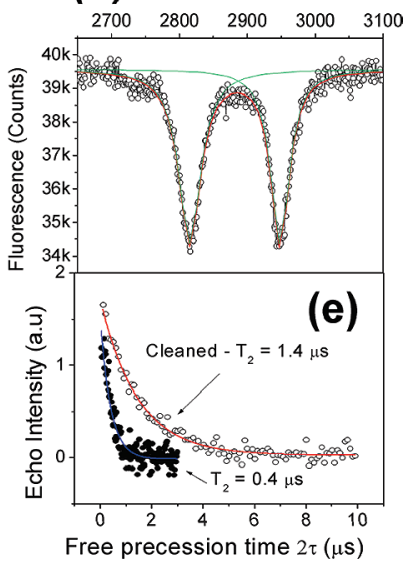

Figure 3. Results on a representative nanocrystal of $7 \mathrm{~nm}$ size. (a) AFM scan and profile of the crystal showing the size. (b) Time trace of fluorescence intensity counts showing a photostable NV center in the $7 \mathrm{~nm}$ nanodiamond. (c) Fluorescence lifetime decay curve showing a lifetime of $14.3 \mathrm{~ns}$. (d) Optically detected magnetic resonance (ODMR) scan showing single spin manipulation. (e) Pulsed ODMR studies of Hahn echo results showing spin coherence properties of a single NV center are preserved in small nanocrystals.

axial symmetry of the center and is expected to be 0 for ideal $C_{3 v}$ NV symmetry. Typical values for $D$ in nanocrystals are close to those found for bulk defects. In most nanocrystals, however, $E$ values significantly deviate from 0 , showing the strain is present. Lattice and symmetry distortions are caused by the strain that arises because of mechanical milling process involved in the production of nanocrystals. In small nanocrystals, these effects are seen prominently, while defects in bulk diamond always shows value of $E=0$. More important than the static spin properties are those associated with spin dynamics. The spatial resolution and field sensitivity in magnetometry application are determined by the ratio of the external field gradient to the electron spin resonance line width. ${ }^{25}$ For applications to magnetic field sensing, it is thus important to have an ESR line as narrow as possible. Assuming the use of spin echoes to remove slow variations, the limiting factor to this line width is the dephasing $\left(T_{2}\right)$ time of the electron spin. In particular, the magnetic field sensitivity is $\Delta B=\pi \hbar / g \mu_{B} \bigvee T_{2}$. The dephasing time has been measured for different defects. Overall, we do find dephasing times ranging from $250 \mathrm{~ns}$ to $1.4 \mu \mathrm{s}$. Surface cleaned diamonds on average do show $T_{2}=1 \mu \mathrm{s}$ (see Figure 3e), which is considerably shorter than measured previously in high purity bulk material, where dephasing times of $T_{2}=350 \mu \mathrm{s}$ have been reported, but comparable to measurements in bulk diamond having a similar nitrogen impurity concentration. ${ }^{26}$ Defect center spin dephasing in diamond is usually limited by mutual spin interactions giving rise to spectral diffusion of the defect spin. This spectral diffusion is driven by a spin bath of impurity spins in the material. The spin bath can be built up by electron or nuclear spins. ${ }^{27,28}$ For NV defects, the spin dephasing time caused by paramagnetic ${ }^{13} \mathrm{C}$ nuclei in natural abundance is some few hundreds of microseconds. The NV defects created in an isotopically enriched ${ }^{12} \mathrm{C}$ diamond show a spin coherence time close to about $2 \mathrm{~ms}^{29}$ The major cause of decoherence in our nanocystals, however, is obviously other spins interacting much more strongly with the NV spin. Prime candidate is substitutional nitrogen, which is contained in nanocrystals in rather large quantities (200 ppm), which is typical for type lb diamond. However, given the small size of the nanodiamonds, only a few $(\sim 18)$ nitrogen atoms are in the nanocrystals. This might not be enough to form an efficient spin bath on their own. Rather, similar to the luminescence properties, in our nanocrystals, we do find a strong impact of the surface on spin dephasing time. Without thorough cleaning, the NV spins in the crystals typically show dephasing times of around a few hundred nanoseconds (see Figure 3e). From the difference of the intrinsic (bulk) dephasing times and these faster decoherence rates, the density of surface spins can be calculated roughly as follows. ${ }^{30}$ One can obtain an estimate of the density of the dephasing spins by applying the Fermi "golden rule" to this situation. If $\Delta \omega_{\text {st }}$ is the static line width of the electron spin resonance transition, then we can take $\pi / \Delta \omega_{\text {st }}$ as the density of states in the center of the line and $\hbar \Delta \omega_{\text {dip }}$ as a mean value for the transition matrix element. We thus have $\left[T_{\mathrm{f}}(\text { center })\right]^{-1} \simeq \pi\left(\Delta \omega_{\text {dip }}\right)^{2} / \Delta \omega_{\text {st. }}$. Away from the center of the line, the density will follow some line shape function. Assuming this to be approximately Lorentzian, we obtain a mean value for the spin flip time $T_{\mathrm{f}}$ given by $T_{\mathrm{f}} \simeq \Delta \omega_{\mathrm{st}} /\left[2 \pi\left(\Delta \omega_{\text {dip }}\right)^{2}\right]$. To relate the measured phase memory time to this spin flip time, a variety of models like the Gauss-Markov or the sudden jump model can be used. For the latter one, the dephasing time is $T_{2}=$ $1.41\left(\Delta \omega_{\text {dip }} / T_{\mathrm{f}}\right)^{-1 / 2}$. In this simple model, the dephasing time depends on the static line width, and the dipolar coupling width is a function of the areal density of paramagnetic defects on the nanodiamond surface. The di- 
polar coupling thus roughly relates to the measured phase memory time as $\Delta \omega_{\text {dip }} \approx\left(\omega_{\text {st }} / T_{2}^{2}\right)^{1 / 3}$. For a $T_{2}$ of 0.5 $\mu \mathrm{s}$, we thus expect a $\Delta \omega_{\text {dip }}$ of $2.7 \mathrm{MHz}$. This results in an areal density of roughly $10 \mu_{B} / \mathrm{nm}^{2}$ on the nanodiamond surface..$^{30}$ For a nanocrystal with $10 \mathrm{~nm}$ diameter, this amounts to about 3000 spins on the surface or approximately 1 spin per $\mathrm{nm}^{2}$. From ESR measurements on nanodiamonds with $30 \mathrm{~nm}$ average diameter, we estimate the surface spin concentration to be roughly 13 spins $/ \mathrm{nm}^{2}$ (refer Materials and Methods).

\section{CONCLUSION}

In conclusion, we have proven that single diamond nanocrystals of $7 \mathrm{~nm}$ diameter show stable NV center luminescence with photophysical properties which are within the expected range. We do not detect a significant reduction in the probability to produce color centers in nanocrystallites other than governed by size reduction. These observations point toward an unchanged structural stability of the NV defect in the small nanocrystals. Earlier calculations showed a tendency of nitrogen in diamond nanocrystallites to be expelled to the crystallite surface. However, those calculations were done on still smaller ( $\emptyset 2.3 \mathrm{~nm}$ ) structure, which might explain the discrepancy with our finding. Given the observed long-term photostability, the photoexcited electron must be stable within the nanocrystals. Because of the high electron mobility in the conduction band of very clean bulk diamond and the negative electron affinity of some crystal surfaces, one might wonder about the physical origin of the stabilization of electrons in nanocrystals. With a concentration of 100 ppm of nitrogen in the material, one has on average on the order of 18 nitrogen atoms per $10 \mathrm{~nm}$ nanocrystals. These on the one hand can provide the extra electron for the NV center minus charge; on the other hand, they can provide traps for the electron after photoexcitation. The smaller the crystal gets, the less excess charges it can provide for the NV center and hence the more likely photobleaching occurs due to photoionization. This size limit obviously is not reached for the present size of nanodiamonds. In bulk samples, NV centers being implanted $2 \mathrm{~nm}$ below the surface showed the same photostability as those being deposited in the bulk material, which indicates that at least surfacerelated effects will allow us to further reduce the size of the nanodiamonds substantially.

\section{MATERIALS AND METHODS}

Materials: In our studies, commercially available diamond nanocrystals (Syp 0.02 van Moppes SA) with a nominally average size of $10 \mathrm{~nm}$ were used. Irradiation was carried out using electrons of about $8 \mathrm{MeV}$ energy. Upon annealing at $700{ }^{\circ} \mathrm{C}$ for about $2 \mathrm{~h}$ in vacuum, the nanodiamonds were boiled in a mixture of sulfuric, nitric, and perchloric acids in a volume ratio (1: 1:1) under reflux at $92{ }^{\circ} \mathrm{C}$ for $24 \mathrm{~h}$ in order to remove graphitic and organic impurities from the surface. The cleaning step involved neutralizing by repeated centrifugation $(8 \times 30 \mathrm{~min}$ at $30000 \mathrm{~g}$ ), discarding the supernatant and suspending the sediment pellet in deionized water. The electron energy and the dosage are the important parameters, while annealing temperature and time can be within a broad window. The PL and spin results discussed in this paper are typical results from the samples after cleaning. This repeated cleaning has helped to improve the spin coherence time by roughly 3.5-fold (see Figure 3e). The improvement in the PL properties, upon cleaning, is difficult to analyze. The PL intensity depends on the orientation of the dipole to detection axis, and the fluorescence lifetime depends on the dipole orientation to the interface.

Sample Preparation: The acid cleaned samples were found to be readily dispersible in water and stable for a long period of time. For electron microscopy studies, a drop of the sample was let to dry onto the carbon grid, and the selected area was imaged. For further studies, a known amount of the nanocrystals was mixed with 0.3 wt \% poly(vinly alcohol) (Aldrich $34,158-4$ ) and spin coated onto a plasma cleaned thin glass coverslip. The AFM-based size measurements of nanodiamonds dispersed on a coverslip (see Figure 1) confirm that the centrifuged material contains a significant fraction of crystals with sizes below $10 \mathrm{~nm}$. The samples prepared in a similar manner were used for fluorescence lifetime studies and spin coherence time investigations.

The Experimental Setup: The studies were performed using a home-built scanning confocal microscope integrated with an AFM (MFP-3D Asylum Research). The nitrogen-vacancy defects in the nanocrystals were excited with a frequency doubled cw Nd:YAG laser (Coherent Compass) focused onto the dispersed sample with a high NA objective (Olympus PlanAPO, NA = 1.35). Luminescence light was collected by the same objective and fil- tered from the excitation light using a dichroic beamsplitter (640 DCXR, Chroma) and long-pass filter (647 LP, Chroma). Photon counting of the filtered light was performed using two avalanche photodiodes (SPQR-14, Perkin-Elmer). Fluorescence lifetime and the autocorrelation histograms were recorded using a TCSPC electronics (PicoHarp 300, PicoQuant GmbH) together with a two channel router controlled by SymPhoTime V 4.0 software. Optically detected magnetic resonance measurements were performed using a commercial microwave source (Rhode \& Schwarz GmbH, SMIQ 03) amplified by a traveling wave tube amplifier (Hughes $8020 \mathrm{H}$ ). Commercially available cantilevers (Olympus OMCL 240-TS) were used in the AFM for recording the topography.

Sample Characterization: The photoluminescent nanodiamonds were characterized in an integrated AFM confocal microscope setup. The confocal microscope detection consists of a Hanbury Brown and Twiss arrangement for measuring photon coincidences. The second-order correlation measurement provides us the information on the number of luminescent defects in a particular nanocrystal. The setup allows us to simultaneously record the topography and the photoluminescence from the nanocrystals. The TCSPC electronics used were also capable of measuring the fluorescence lifetime of the desired individual nanocrystals. Furthermore, the setup was synchronized such that it was able to record the fluorescence lifetime image of the nanocrystals together with the topography. The spin properties of the defects in selected nanocrystals were investigated by optically detected magnetic resonance.

Optically Detected Magnetic Resonance: Single defects can be isolated and individually addressed using confocal microscopy. Two out of 16 electrons of the NV defect are unpaired, forming a triplet spin system. Spin-spin interactions of the electron split the energy levels with magnetic quantum numbers $m_{\mathrm{s}}=0$ and 1 by about $2.88 \mathrm{GHz}$. The degeneracy of $m_{\mathrm{s}}= \pm 1$ states that arise because of the $C_{3 v}$ symmetry can be lifted further by applying an external magnetic field. Under optical illumination, spinselective relaxations lead to an efficient optical pumping of the system into a $m_{\mathrm{s}}=0$ state that allows fast $(250 \mathrm{~ns})$ polarization of the spin. The spin state of a single NV defect can be manipulated by applying resonant microwave fields. The difference in 
the intersystem crossing rates leads to a situation that the fluorescence from the $m_{\mathrm{s}}=0$ state scatters $30 \%$ more photons than from the $m_{\mathrm{s}}= \pm 1$ states. Thus, the spin state of a single defect can be monitored and manipulated optically.

CW and Pulsed ODMR Experiments: At constant bias magnetic field of few gauss, the continuous wave (CW) ODMR spectrum was obtained by sweeping the microwave frequency and monitoring the fluorescence intensity. When the frequency matches the resonance, we observe a decrease in fluorescence as explained above. A representative spectrum is shown in Figure $3 d$. The obtained spectrum is often power broadened. To retrieve the true line widths, coherent manipulation techniques were performed. The NV spin is polarized using a brief laser pulse, followed by a pulse of resonant microwave frequency of varying pulse lengths. The microwave pulse transfers the population between $m_{s}=0$ and $m_{s}= \pm 1$ levels. This results in Rabi oscillations, from which we define a $\pi / 2$ and the $\pi$ pulses. Hahn echo experiments are used to get the spin dephasing times $\left(T_{2}\right)$. The Hahn echo sequence consists of a brief laser pulse followed by a $\pi / 2-\tau-\pi-\tau-\pi / 2$ microwave pulse trains. The free precession time $\tau$ is varied, and then population at $m_{\mathrm{s}}=0$ state is monitored. A simple fit to an expression given by $P\left(m_{\mathrm{s}=0}\right)=\exp (-2 \tau /$ $\left.T_{2}\right)^{2}$ gives $T_{2}$ spin dephasing time of the single spin under investigation.

Calculations of the Surface Spin Concentration: The ESR characterization of the surface spins were carried out in a Bruker Elexsys W Band spectrometer. The microwave frequency used was $94 \mathrm{GHz}$ at 3.3 T magnetic field. The result of the ESR spectrum is shown in the Supporting Information. We assume that the shape of the nanodiamond is a sphere with diameter $d=30 \mathrm{~nm}$. The volume $V$ and the surface area $S$ are $V=\pi d^{3} / 6=14137.2 \mathrm{~nm}^{3}$ and $S=$ $\pi d^{2} / 4=2826 \mathrm{~nm}^{2}$. The mass of the nanodiamond is $m=\rho V=$ $4.95 \times 10^{-17} \mathrm{~g}$, and it consists of $\left.N=m N_{\mathrm{A}} / A_{\mathrm{C}}\right)=4.95 \times 10^{-17} \times$ $6.022 \times 10^{23} / 12.01=2.48 \times 10^{6}$ atoms, where $A_{C}$ is the carbon atomic mass and $N_{\mathrm{A}}$ is Avogadro's number (we neglect the nitrogen impurities in this calculation). From EPR measurements on these diamonds before they were milled, we obtained a nitrogen concentration (P1 center) of $200 \mathrm{ppm}$. Thus, the number of nitrogen atoms in a nanodiamond is $2 \times 10^{-4} \times 2.48 \times 10^{6}=$ 496. They cause the small peaks in the ESR spectrum, where the large signal comes from the surface spins. The ratio of the integrated spectral lines is (nitrogen to surface spins) $I_{N} / I_{S}=1: 76$, and we can estimate the number of the surface spins to be $4796 \times$ $76=37696$. Here we assumed that both types of spins have the same saturation behavior, which is not really the case. The surface spin concentration is calculated to be about $37696 / 2826 \approx$ 13 spins $/ \mathrm{nm}^{2}$.

Acknowledgment. Financial support from the European Commission through the project "Nano4Drugs" (contract LSHB-2005CT-019102) and the Volkswagenstiftung as well as NIH under Grant 1R01GM084712-01, CFDA No. 93.859) is acknowledged.

Supporting Information Available: Figure showing the ESR spectrum of $30 \mathrm{~nm}$ nanodiamonds at room temperature. Experimental parameters: Microwave frequency: $94.08 \mathrm{GHz}$, microwave power $0.2 \mathrm{~mW}$, field modulation amplitude $0.1 \mathrm{mT}$, modulation frequency $100 \mathrm{kHz}$. This material is available free of charge via the Internet at http://pubs.acs.org.

\section{REFERENCES AND NOTES}

1. Chang, Y. R.; Lee, H. Y.; Chen, K.; Chang, C. C.; Tsai, D. S.; Fu, C. C.; Lim, T. S.; Tzeng, Y. K.; Fang, C. Y.; Han, C. C.; Chang, H. C.; Fann, W. Mass Production and Dynamic Imaging of Fluorescent Nanodiamonds. Nat. Nanotechnol. 2008, 3, 284-288.

2. Fu, C. C.; Lee, H. Y.; Chen, K.; Lim, T. S.; Wu, H. Y.; Lin, P. K.; Wei, P. K.; Tsao, P. H.; Chang, H. C.; Fann, W. Characterization and Application of Single Fluorescent Nanodiamonds as Cellular Biomarkers. Proc. Natl. Acad. Sci. U.S.A. 2007, 104, 727-732.

3. Krueger, A. Diamond Nanoparticles: Jewels for Chemistry and Physics. Adv. Mater. 2008, 20, 2445-2449.

4. Huang, H. J.; Pierstorff, E.; Osawa, E.; Ho, D. ProteinMediated Assembly of Nanodiamond Hydrogels into a
Biocompatible and Biofunctional Multilayer Nanofilm. ACS Nano 2008, 2, 203-212.

5. Park, Y. S.; Cook, A. K.; Wang, H. L. Cavity QED with Diamond Nanocrystals and Silica Microspheres. Nano Lett. 2006, 6, 2075-2079.

6. Schietinger, S.; Schroder, T.; Benson, O. One-by-One Coupling of Single Defect Centers in Nanodiamonds to High-Q Modes of an Optical Microresonator. Nano Lett. 2008, 8, 3911-3915.

7. Schrand, A. M.; Huang, H. J.; Carlson, C.; Schlager, J. J.; Osawa, E.; Hussain, S. M.; Dai, L. M. Are Diamond Nanoparticles Cytotoxic? J. Phys. Chem. B 2007, 111, 2-7.

8. Lam, R.; Chen, M.; Pierstorff, E.; Huang, H.; Osawa, E. J.; Ho, D. Nanodiamond-Embedded Microfilm Devices for Localized Chemotherapeutic Elution. ACS Nano 2008, 2, 2095-2102.

9. Michalet, X.; Pinaud, F. F.; Bentolila, L. A.; Tsay, J. M.; Doose, S.; Li, J. J.; Sundaresan, G.; Wu, A. M.; Gambhir, S. S.; Weiss, S. Quantum Dots for Live Cells, In Vivo Imaging, and Diagnostics. Science 2005, 307, 538-544.

10. Cao, L.; Wang, X.; Meziani, M. J.; Lu, F. S.; Wang, H. F.; Luo, P. J. G.; Lin, Y.; Harruff, B. A.; Veca, L. M.; Murray, D.; Xie, S. Y.; Sun, Y. P. Carbon Quantum Dots for Multiphoton Bioimaging. J. Am. Chem. Soc. 2007, 129, 11318-11319.

11. Beveratos, A.; Brouri, R.; Gacoin, T.; Poizat, J. P.; Grangier, P. Nonclassical Radiation from Diamond Nanocrystals. Phys. Rev. A 2001, 64, 61802.

12. Kurtsiefer, C.; Mayer, S.; Zarda, P.; Weinfurter, H. Stable Solid-State Source of Single Photons. Phys. Rev. Lett. 2000, 85, 290-293.

13. Wu, E.; Jacques, V.; Zeng, H. P.; Grangier, P.; Treussart, F.; Roch, J. F. Narrow-Band Single-Photon Emission in the Near Infrared for Quantum Key Distribution. Opt. Express 2006, 14, 1296-1303.

14. Balasubramanian, G.; Chan, I. Y.; Kolesov, R.; Al-Hmoud, M.; Tisler, J.; Shin, C.; Kim, C.; Wojcik, A.; Hemmer, P. R.; Krueger, A.; Hanke, T.; Leitenstorfer, A.; Bratschitsch, R.; Jelezko, F.; Wrachtrup, J. Nanoscale Imaging Magnetometry with Diamond Spins under Ambient Conditions. Nature 2008, 455, 648-651.

15. Hartl, A.; Schmich, E.; Garrido, J. A.; Hernando, J.; Catharino, S. C. R.; Walter, S.; Feulner, P.; Kromka, A.; Steinmuller, D.; Stutzmann, M. Protein-Modified Nanocrystalline Diamond Thin Films for Biosensor Applications. Nat. Mater. 2004, 3, 736-742.

16. Rubio-Retama, J.; Hernando, J.; Lopez-Ruiz, B.; Hartl, A.; Steinmuller, D.; Stutzmann, M.; Lopez-Cabarcos, E.; Garrido, J. A. Synthetic Nanocrystalline Diamond as a Third-Generation Biosensor Support. Langmuir 2006, 22, 5837-5842.

17. Yang, W. S.; Auciello, O.; Butler, J. E.; Cai, W.; Carlisle, J. A.; Gerbi, J.; Gruen, D. M.; Knickerbocker, T.; Lasseter, T. L.; Russell, J. N.; Smith, L. M.; Hamers, R. J. DNA-Modified Nanocrystalline Diamond Thin-Films as Stable, Biologically Active Substrates. Nat. Mater. 2003, 2, 63.

18. Wu, E.; Rabeau, J. R.; Roger, G.; Treussart, F.; Zeng, H.; Grangier, P.; Prawer, S.; Roch, J. F. Room Temperature Triggered Single-Photon Source in the Near Infrared. New J. Phys. 2007, 9, 434.

19. Osawa, E. Monodisperse Single Nanodiamond Particulates. Pure Appl. Chem. 2008, 80, 1365-1379.

20. Smith, B. R.; Inglis, D. W.; Sandnes, B.; Rabeau, J. R.; Zvyagin, A. V.; Gruber, D.; Noble, C. J.; Vogel, R.; Sawa, E.; Plakhotnik, T. Five-Nanometer Diamond with Luminescent Nitrogen-Vacancy Defect Centers. Small DOI: 10.1002/smll.200801802

21. Wrachtrup, J.; Jelezko, F. Processing Quantum Information in Diamond. J. Phys.: Condens. Matter 2006, 18, S807-S824.

22. Campbell, B.; Mainwood, A. Radiation Damage of Diamond by Electron and Gamma Irradiation. Phys. Status Solidi A 2000, 181, 99-107.

23. Batalov, A.; Zierl, C.; Gaebel, T.; Neumann, P.; Chan, I. Y.; Balasubramanian, G.; Hemmer, P. R.; Jelezko, F.; Wrachtrup, 
J. Temporal Coherence of Photons Emitted by Single Nitrogen-Vacancy Defect Centers in Diamond Using Optical Rabi-Oscillations. Phys. Rev. Lett. 2008, 100, 77401.

24. Lukosz, W.; Kunz, R. E. Fluorescence Lifetime of Magnetic and Electric Dipoles near a Dielectric Interface. Opt. Commun. 1977, 20, 195-199.

25. Maze, J. R.; Stanwix, P. L.; Hodges, J. S.; Hong, S.; Taylor, J. M.; Cappellaro, P.; Jiang, L.; Dutt, M. V. G.; Togan, E.; Zibrov, A. S.; Yacoby, A.; Walsworth, R. L.; Lukin, M. D. Nanoscale Magnetic Sensing with an Individual Electronic Spin in Diamond. Nature 2008, 455, 644-647.

26. Jelezko, F.; Gaebel, T.; Popa, I.; Gruber, A.; Wrachtrup, J. Observation of Coherent Oscillations in a Single Electron Spin. Phys. Rev. Lett. 2004, 92, 76401.

27. Hanson, R.; Dobrovitski, V. V.; Feiguin, A. E.; Gywat, O.; Awschalom, D. D. Coherent Dynamics of a Single Spin Interacting with an Adjustable Spin Bath. Science 2008, 320, 352-355.

28. Maze, J. R.; Taylor, J. M.; Lukin, M. D. Electron Spin Decoherence of Single Nitrogen-Vacancy Defects in Diamond. Phys. Rev. B 2008, 78, 94303.

29. Balasubramanian, G.; Neumann, P.; Twitchen, D.; Markham, M.; Kolesov, R.; Mizuoschi, N.; Isoya, J.; Achard, J.; Beck, J.;

Tisler, J.; Jacques, V.; Hemmer, P. R.; Jelezko, F.; Wrachtrup, J. Ultralong Spin Coherence Time in Isotopically Engineered Diamond. Nat. Mater. 2009, 8, 383-387.

30. Mims, W. B. In Electron Paramagnetic Resonance; Geschwind, S., Ed.; Plenum Press: New York, London, 1972. 